\title{
Abrasión intraósea por sutura según el ángulo del túnel transóseo en huella del manguito rotador
}

\section{Intraosseous Suture Abrasion according to the Angle of the Transosseous Tunnel in Rotator Cuff Footprint}

\author{
Julio José Contreras ${ }^{10}$ Rodrigo Liendo ${ }^{2(-)}$ Andrés Meza $^{3(1)}$ Francisco Soza ${ }^{2}$ \\ 1 Unidad de Hombro y Codo, Instituto Traumatológico, Santiago de \\ Chile, Chile \\ 2 Pontificia Universidad Católica de Chile, Santiago de Chile, Chile \\ 3 Ortopedia y Traumatología, Hospital Claudio Vicuña, San Antonio, \\ Valparaíso, Chile \\ Address for correspondence Julio José Contreras Fernández, MD, \\ Unidad de Hombro y Codo,, Instituto Traumatológico, San Martín 771, \\ Santiago de Chile, Chile (e-mail: Juliocontrerasmd@gmail.com). \\ Rev Chil Ortop Traumatol 2021;62(3):e168-e173.
}

\section{Resumen \\ Palabras Claves \\ - manguito de los rotadores \\ - sutura \\ - técnicas de sutura \\ - traumatismos de los tendones \\ - tendones \\ - desgaste óseo \\ - abrasión por sutura \\ - abrasión cíclica \\ Objetivo Comparar el desgaste óseo generado por la abrasión de una carga cíclica entre túneles clásicos oblicuos y perpendiculares. Nuestra hipótesis es la de que el túnel oblicuo presenta un menor desgaste óseo por abrasión cíclica comparado con el túnel perpendicular. \\ Métodos Ocho hombros congelados de cordero fueron usados para el estudio biomecánico. En cada húmero proximal, dos túneles (oblicuo y perpendicular) fueron generados en la tuberosidad mayor. Se utilizó un sistema de tracción cíclica para traccionar hacia atrás y adelante una sutura trenzada en tensión a través del túnel, midiendo la distancia entre la entrada y la salida de la sutura en el túnel antes y después del proceso de ciclado como medida de perdida de tensión de la sutura. El resultado principal es el cambio de la distancia entre la entrada y la salida de la sutura en el túnel después del ciclado para estimar el desgaste óseo dentro del túnel. Para el análisis estadístico, se utilizó la prueba $U$ de Mann-Whitney. Se consideraron significativos valores de $p<0,05$. \\ Resultados Los túneles perpendiculares tuvieron un 23,24 $\pm 7,44 \%$ de pérdida de longitud, y los túneles oblicuos, 7,76 $\pm 4,32 \%$. La diferencia de pérdida de longitud fue significativa $(p=0,0003)$. \\ Conclusión La abrasión ósea generada por el movimiento cíclico de la sutura en el túnel transóseo está influenciada por la geometría del túnel. El desgaste óseo es menor en un túnel oblicuo comparado con un túnel perpendicular. \\ Nivel de Evidencia Estudio de ciencia básica.}

recibido

28 de agosto de 2020

aceptado

06 de agosto de 2021
DOI https://doi.org/

$10.1055 / \mathrm{s}-0041-1740095$ ISSN $0716-4548$. (c) 2021. Sociedad Chilena de Ortopedia y Traumatologia. All rights reserved.

This is an open access article published by Thieme under the terms of the Creative Commons Attribution-NonDerivative-NonCommercial-License, permitting copying and reproduction so long as the original work is given appropriate credit. Contents may not be used for commercial purposes, or adapted, remixed, transformed or built upon. (https://creativecommons.org/ licenses/by-nc-nd/4.0/)

Thieme Revinter Publicações Ltda., Rua do Matoso 170, Rio de Janeiro, RJ, CEP 20270-135, Brazil 


\section{Abstract}

\section{Keywords}

- rotator cuff

- suture

- suture techniques

- tendon injuries

- tendons

- bone tunnel wear

- suture abrasion

- cyclic abrasion
Objective To compare the bone wear generated by the abrasion of a cyclic load between classic oblique and perpendicular tunnels. Our hypothesis is that the oblique tunnel is submitted to less cyclic abrasion bone wear compared with the perpendicular tunnel.

Methods Eight fresh-frozen lamb shoulders were used for biomechanical testing. In each proximal humerus, two tunnels (one oblique and one perpendicular) were drilled at the greater tuberosity. We used a cyclic traction system to pull back and forth a braided suture under tension through the tunnel, measuring the distance between the entry and exit points of the suture within the tunnel before and after the cyclic process to release the tension in the suture. The main outcome was the percentage of change in the distance between the entry and exit points of the suture within the tunnel before and after cyclic abrasion to estimate the degree of bone wear inside the tunnel. For the statistical analysis, the Mann-Whitney $U$ test was used. Values of $p<0.05$ were considered significant.

Results The perpendicular bone tunnels had $23.24 \pm 7.44 \%$ decrease in length, and the oblique bone tunnels, $7.76 \pm 4.32 \%$. The difference in the decrease in length was significant $(p=0.0003)$.

Conclusion The bone abrasion caused by the cyclical movement of the suture in the bone tunnel was influenced by the shape of the tunnel. Bone wear was lower with an oblique tunnel compared with a perpendicular tunnel.

Level of Evidence Basic Science Study.

\section{Introducción}

Las roturas del manguito rotador son una causa muy común de dolor y de Odéficit de la función del hombro. ${ }^{1}$ Este tipo de roturas se estima tiene una prevalencia de un $20,7 \%$ en la población general, que aumenta con la edad. ${ }^{1,2}$ La reparación de una rotura de manguito rotador alivia el dolor y mejora la función. $^{3}$

Idealmente, la reparación debe tener la fuerza de compresión suficiente para minimizar la separación y mantener una estabilidad mecánica hasta que la cicatrización esté completa. ${ }^{4}$ Por este motivo, la reparación además debe ser capaz de soportar una carga cíclica fisiológica. ${ }^{5}$

La forma de tratamiento más común de una rotura de manguito rotador es la reinserción con anclas, en modalidad abierta o artroscópica, con adecuados resultados funcionales: ${ }^{6-11} \sin$ embargo, todavía existe una alta tasa de falla en la reparación. ${ }^{12-14}$ Los factores clínicos más importantes que influyen en el proceso de cicatrización son el tamaño de la rotura, el grado de degeneración grasa de los músculos, la calidad del tejido, y la edad del paciente. $^{13-18}$ Muchos factores biomecánicos contribuyen al proceso de cicatrización y, así, para evitar la falla en la reparación, e incluyen el área y la presión de contacto del tendón a la huella ${ }^{19}$ y la tensión de la sutura en el movimiento en la interfase tendón-huella. ${ }^{20}$

Los sistemas de reparación con anclas son ampliamente utilizados, pero tienden a fallar en su fijación en hueso osteoporótico, lo que lleva a una disminución de la presión de contacto en la huella. ${ }^{21}$ Para disminuir la tasa de rerrotura, ${ }^{22}$ de avulsión de las anclas, disminuir costos, ${ }^{23-25}$ y mejorar la cicatrización del tendón, ${ }^{26,27}$ se han desarrollado nuevos métodos de reparación con técnicas clásicas de suturas transóseas.

Las técnicas de fijación transósea pueden ser realizadas en forma artroscópica o abierta, y han demostrado su eficiencia en la reparación de manguito rotador. ${ }^{28}$ En la técnica clásica abierta, se realizaba el túnel transóseo con grandes agujas oblicuas, ${ }^{29}$ que se han tratado de reproducir bajo artroscopia, ${ }^{30}$ pero los sistemas más popularizados se basan en técnicas artroscópicas que crean túneles transóseos perpendiculares, intersectando un túnel medial en la huella con otro $1,5 \mathrm{~cm}$ bajo la punta de la tuberosidad. ${ }^{31}$ El paso de una sutura por el hueso agrega una zona de falla debido a la abrasión en carga cíclica que debe ser considerada. ${ }^{5}$

Dependiendo de la geometría del túnel transóseo, podría cambiar la abrasión sobre el hueso esponjoso y cortical durante la carga cíclica. El objetivo de este estudio biomecánico es comparar el desgaste óseo generado por la abrasión de una carga cíclica entre túneles clásicos oblicuos y perpendiculares. Nuestra hipótesis es la de que el túnel oblicuo presenta un menor desgaste óseo por abrasión cíclica comparado con el túnel perpendicular.

\section{Materiales y Métodos}

\section{Modelo Animal}

Ocho hombros de cordero (Ovis orientalis aries) fueron descongelados a temperatura ambiente y disecados para las pruebas biomecánicas. Todo el tejido blando alrededor 
del húmero proximal fue removido para identificar la tuberosidad mayor. No se encontraron anormalidades en el manguito rotador en ningún espécimen. Todos las piezas fueron irrigadas con solución salina para prevenir la deshidratación del tejido. No hubo procesamiento de animales vivos; todos los especímenes se consiguieron de una empresa de manejo de animales (Simunovic Ltda.).

\section{Diseño del Túnel}

En la tuberosidad mayor, se realizaron 2 túneles de $2,5 \mathrm{~mm}$ de diámetro, separados por $10 \mathrm{~mm}$. Se utilizó un compás diseñado para realizar un túnel perpendicular y otro túnel oblicuo (radio de $15 \mathrm{~mm}$ ) en cada espécimen. En ambos túneles, la entrada estaba $10 \mathrm{~mm}$ lateral al borde de la tuberosidad, y la salida, $10 \mathrm{~mm}$ medial al borde de la tuberosidad, que corresponde a la zona medial de la huella del infraespinoso en el cordero (-Figura 1).

\section{Modelo de Microabrasión Cíclica}

Se utilizó un motor de ciclado hecho a medida que permitía traccionar la sutura atrás y adelante a una frecuencia de $2,5 \mathrm{~Hz}$ con una excursión de $5 \mathrm{~cm}$ (velocidad de $150 \mathrm{~cm} /$ minuto), con una carga de $10 \mathrm{~N}$ (-Figura 2). Estos parámetros fueron descritos en estudios similares, ${ }^{32,33}$ y simulan cargas fisiológicas en actividades de la vida diaria. Respecto al eje de tracción de las suturas, este es en $90^{\circ}$ en relación a la superficie ósea del orificio del túnel transóseo (-Fig. 3 ).

El test de abrasión se realizó con una sutura con núcleo central de polietileno y recubierta de hebras múltiples trenzadas de alto peso molecular (USP No. 2; FiberWire, Arthrex, Naples, FL, EEUU). La distancia entre la entrada y salida de la sutura en el túnel fue medida antes y después de 1.400 ciclos con un calibre digital (con $0,1 \mathrm{~cm}$ de resolución). El diámetro del orificio del hueso cortical no fue medido.

\section{Resultado Principal}

La medición principal es el porcentaje de cambio en la longitud de la sutura que está dentro del túnel oblicuo y
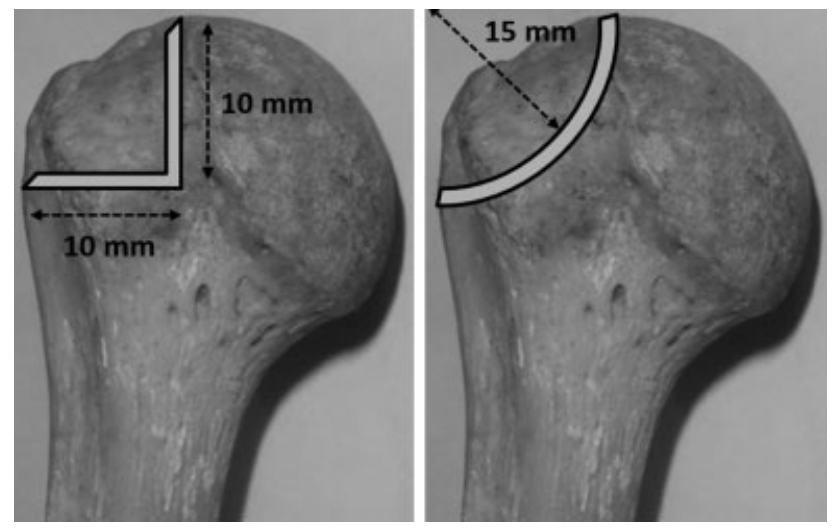

Fig. 1 Diseño de los túneles. A izquierda, el túnel perpendicular; a derecha, el túnel oblicuo (clásico). Se utilizó un compás diseñado para realizar un túnel perpendicular y otro túnel oblicuo (radio de $15 \mathrm{~mm}$ ) en cada espécimen. En ambos túneles, la entrada estaba $10 \mathrm{~mm}$ lateral al borde de la tuberosidad, y la salida, $10 \mathrm{~mm}$ medial al borde de la tuberosidad.

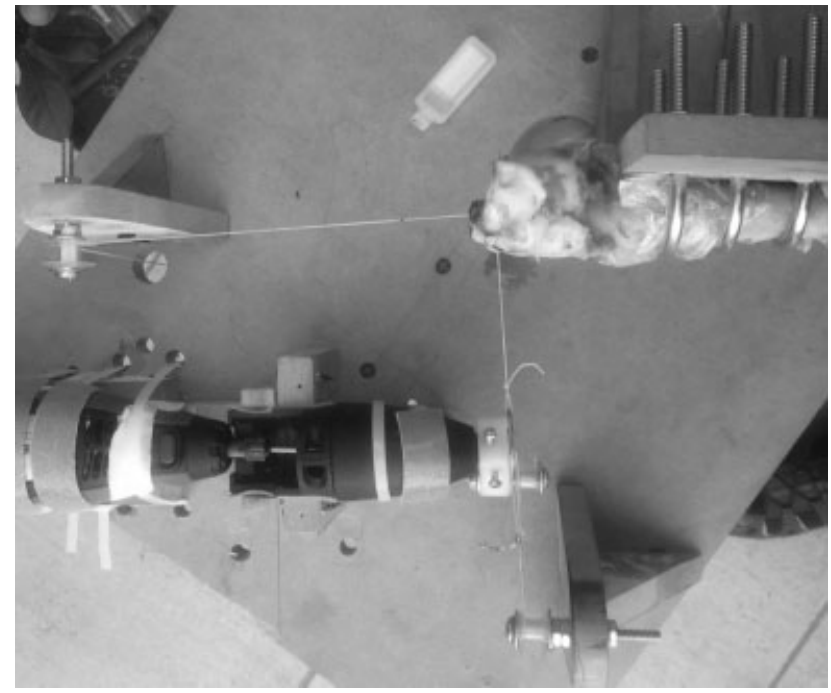

Fig. 2 Modelo hecho a medida para abrasión cíclica. Se utilizó un motor de ciclado hecho a medida que permitía traccionar la sutura atrás y adelante. El húmero proximal fue fijado con una abrazadera, y se aseguró la perpendicularidad de la sutura al momento del ensayo.

perpendicular antes y después del ciclo de microabrasión como estimación del grado de desgaste del túnel óseo.

\section{Análisis Estadístico}

Para el análisis estadístico, considerando un número bajo de datos, se realizó el test no paramétrico U de Mann-Whitney para evaluar la variable estudiada. Todos los datos fueron analizados usando el programa STATA (StataCorp LLC, College Station, TX, EEUU), versión 16. Se estableció la significancia estadística en $p<0,05$ a dos colas.

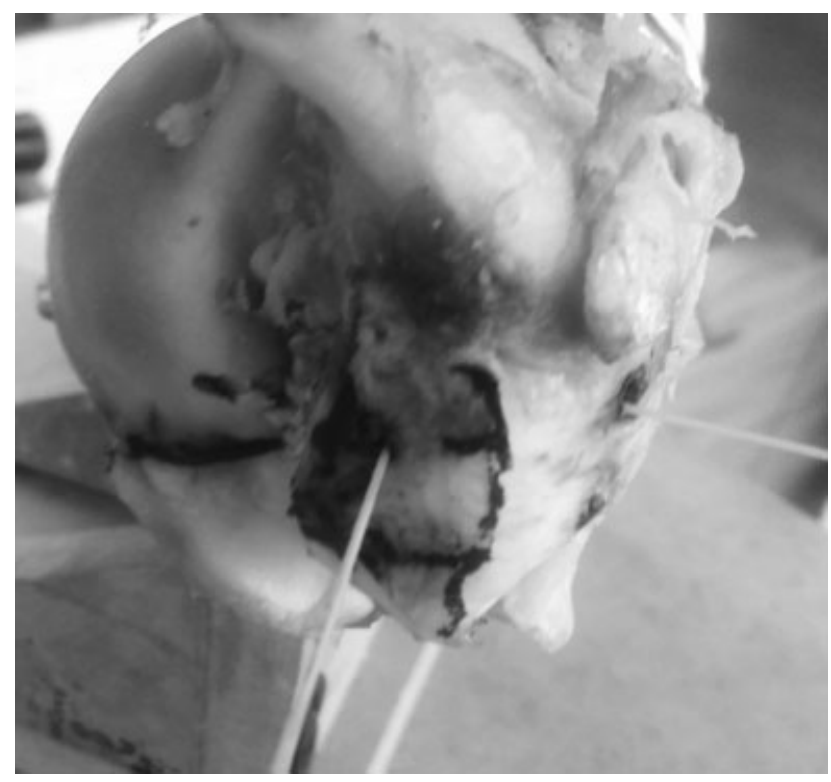

Fig. 3 Disección de la tuberosidad mayor y preparación de los túneles. En esta figura, se observa que todo el tejido blando alrededor del húmero proximal fue removido para identificar la tuberosidad mayor y poder diseñar los túneles. 


\section{Resultados}

Los túneles perpendiculares tenían una longitud preciclado de $1,93 \pm 0,17 \mathrm{~cm}, \mathrm{y}$ una longitud postciclado de $1,48 \pm 0,22 \mathrm{~cm}$ $(23,24 \pm 7,44 \%$ de disminución de longitud). Los túneles oblicuos tenían una longitud preciclado de $1,83 \pm 0,15 \mathrm{~cm}, \mathrm{y}$ una longitud postciclado de $1,69 \pm 0,14 \mathrm{~cm}(7,76 \pm 4,32 \%$ de disminución de longitud) (-Tabla 1). La diferencia entre la disminución de la longitud de los túneles oblicuos y perpendiculares fue significativa $(p=0,0003)$.

\section{Discusión}

El principal resultado de este estudio es que la longitud del túnel intraóseo disminuye después del ciclado como resultado del desgaste óseo del hueso esponjoso causado por el movimiento de la sutura. En el caso de los túneles oblicuos, esto ocurre tres veces menos en comparación con los túneles perpendiculares.

La reparación del manguito rotador requiere de adecuada fuerza de fijación inicial, con un mínimo de pérdida de contacto de la huella hasta que se complete la cicatrización. 4,34 Adicionalmente, la reparación debe resistir una carga cíclica en el tiempo. ${ }^{5}$ Los factores que contribuyen a la reparación son una adecuada calidad del tejido, la presión y el área de contacto, ${ }^{19}$ y el movimiento de la interfase tendón-huella. ${ }^{20}$ Numerosos estudios ${ }^{35-38}$ han demostrado que la presión en la interfase tendón-huella, determinada como la tensión de la sutura, es beneficiosa para la cicatrización. Aunque la presión ideal para la reparación del manguito rotador no esté establecida, su impacto clínico en modelos de variación es incierto. ${ }^{19,27,39}$

Existen varios estudios biomecánicos que demuestran que las reparaciones con técnicas transóseas logran una excelente presión a nivel de la huella, ${ }^{26,27}$ mayor resistencia al fallo, y menor movimiento de la interfase tendón-huella comparadas con técnicas con anclas. ${ }^{20,40}$ Existen series clínicas ${ }^{22}$ que han demostrado bajas tasas de rerotura (6\%) utilizando técnicas transóseas.

La reparación artroscópica intenta replicar las técnicas abiertas, incluso la técnica transósea con diferentes configuraciones de anclas. La transición de técnicas transóseas abiertas a técnica artroscópica no es fácil, dada la dificultad de realizar los túneles transóseos. Puede realizarse un túnel lateral directo con salida a la huella medial, pero presenta la dificultad de la cercanía del punto de entrada con el nervio axilar; ${ }^{41}$ para alejarse de esta zona, es factible utilizar una aguja oblicua, pero es más complejo planificar la salida a nivel de la huella. ${ }^{30}$ Es por esto que la técnica más extendida se basa en un sistema mecánico que

Tabla 1 Abrasión de los túneles óseos estimada como el porcentaje de cambio de longitud de cada configuración de túnel

\begin{tabular}{|l|l|l|l|l|}
\hline Ángulo del túnel & & & & \\
\hline Perpendicular & Preabrasión (cm) & Postabrasión (cm) & $\Delta$ Abrasión & Porcentaje \\
\hline & 2,2 & 1,8 & 0,4 & $18,2 \%$ \\
\hline & 1,8 & 1,3 & 0,5 & $27,8 \%$ \\
\hline & 2 & 1,4 & 0,6 & $30,0 \%$ \\
\hline & 1,9 & 1,65 & 0,25 & $13,2 \%$ \\
\hline & 2,1 & 1,6 & 0,5 & $23,8 \%$ \\
\hline & 1,9 & 1,5 & 0,4 & $21,1 \%$ \\
\hline & 1,8 & 1,5 & 0,3 & $16,7 \%$ \\
\hline & 1,7 & 1,1 & 0,6 & $35,3 \%$ \\
\hline Promedio & 1,93 & 1,48 & 0,44 & $23,24 \%$ \\
\hline Oblicuo & 0,17 & 0,22 & 0,13 & 7,44 \\
\hline & Preabrasión (cm) & Postabrasión (cm) & $\Delta$ Abrasión & Porcentaje \\
\hline & 1,6 & 1,5 & 0,1 & $6,3 \%$ \\
\hline & 1,85 & 1,7 & 0,15 & $8,1 \%$ \\
\hline & 1,9 & 1,8 & 0,1 & $5,3 \%$ \\
\hline & 1,9 & 1,6 & 0,3 & $15,8 \%$ \\
\hline & 1,6 & 1,5 & 0,1 & $6,3 \%$ \\
\hline & 1,9 & 1,8 & 0,1 & $5,3 \%$ \\
\hline & 1,9 & 1,85 & 0,05 & $2,6 \%$ \\
\hline & 2 & 1,75 & 0,25 & $12,5 \%$ \\
\hline & 1,83 & 1,69 & 0,14 & 7,76 \\
\hline & 0,15 & 0,14 & 0,09 & 4,32 \\
\hline & & & & \\
\hline & & & & \\
\hline
\end{tabular}

Note: $\Delta$ : diferencia. 
logra intersectar túneles perpendiculares apuntando a la zona de la huella requerida. ${ }^{23,42}$

En la práctica clínica, existió una transición en el uso de suturas monofilamento o trenzadas de poliéster a suturas trenzadas de materiales mixtos (polyblend). Kowalsky et al. ${ }^{5}$ estudiaron las propiedades abrasivas de diferentes tipos de suturas sobre el tendón y hueso, y demostraron que el desgaste óseo no era dependiente del tipo de sutura. Sin embargo, se necesitan más estudios para determinar el impacto real del tipo de sutura y la abrasión en modelos de túnel óseo.

El presente estudio evaluó propiedades biomecánicas relacionadas con la abrasión cíclica de un túnel óseo en un modelo animal. Se seleccionó el hombro de cordero por poseer características anatómicas y funcionales equivalentes a las del tendón supraespinoso de humanos. ${ }^{43}$ Sin embargo, queda por determinar si las diferencias interespecie pueden afectar la reparación del manguito rotador en humanos. El presente estudio tiene muchas limitaciones. El efecto de la abrasión intraósea busca evaluar el efecto aislado del ángulo del túnel transóseo con un vector de tracción de $90^{\circ}$ respecto a la superficie ósea; sin embargo, un modelo ideal consideraría la realización de una reparación transósea de una rotura simulada del manguito rotador y realizar una tracción cíclica del tendón en cuestión. La densidad ósea del húmero proximal de cordero es distinta a la del humano de edad media, por lo que la variabilidad interespecie no es descartable. Los cadáveres humanos pueden representar mejor el resultado clínico, aunque al ser estudios ex vivo, no hay información sobre la cicatrización.

Finalmente, podemos concluir que la abrasión intraósea generada por el movimiento cíclico de una sutura en un túnel transóseo está influenciada por la geometría del túnel (ángulo). El desgaste óseo es menor en un túnel oblicuo comparado con un túnel perpendicular.

\section{Conflicto de Intereses}

Los autores no tienen conflicto de intereses que declarar.

\section{Agradecimientos}

A nuestra familia, por el constante apoyo en nuestra labor de investigación.

\section{Referencias}

1 Reilly P, Macleod I, Macfarlane R, Windley J, Emery RJ. Dead men and radiologists don't lie: a review of cadaveric and radiological studies of rotator cuff tear prevalence. Ann R Coll Surg Engl 2006; 88(02):116-121. Doi: $10.1308 / 003588406 \times 94968$

2 Yamamoto A, Takagishi K, Osawa T, et al. Prevalence and risk factors of a rotator cuff tear in the general population. J Shoulder Elbow Surg 2010;19(01):116-120. Doi: 10.1016/j.jse.2009.04.006

3 Vaishnav S, Millett PJ. Arthroscopic rotator cuff repair: scientific rationale, surgical technique, and early clinical and functional results of a knotless self-reinforcing double-row rotator cuff repair system. J Shoulder Elbow Surg 2010;19(2, Suppl):83-90. Doi: $10.1016 /$ j.jse.2009.12.012

4 Gerber C, Schneeberger AG, Beck M, Schlegel U. Mechanical strength of repairs of the rotator cuff. J Bone Joint Surg $\mathrm{Br}$ 1994;76(03):371-380
5 Kowalsky MS, Dellenbaugh SG, Erlichman DB, Gardner TR, Levine $\mathrm{WN}$, Ahmad CS. Evaluation of suture abrasion against rotator cuff tendon and proximal humerus bone. Arthroscopy 2008;24(03): 329-334. Doi: 10.1016/j.arthro.2007.09.011

6 Gartsman GM, Khan M, Hammerman SM. Arthroscopic repair of full-thickness tears of the rotator cuff. J Bone Joint Surg Am 1998; 80(06):832-840

7 Burkhart SS, Danaceau SM, Pearce CE Jr. Arthroscopic rotator cuff repair: Analysis of results by tear size and by repair techniquemargin convergence versus direct tendon-to-bone repair. Arthroscopy 2001;17(09):905-912. Doi: 10.1053/jars.2001.26821

8 Murray TF Jr, Lajtai G, Mileski RM, Snyder SJ. Arthroscopic repair of medium to large full-thickness rotator cuff tears: outcome at 2to 6-year follow-up. J Shoulder Elbow Surg 2002;11(01):19-24. Doi: $10.1067 / \mathrm{mse} .2002 .120142$

9 Flurin P-H, Landreau P, Gregory T, et al. Cuff Integrity After Arthroscopic Rotator Cuff Repair: Correlation With Clinical Results in 576 Cases. Arthroscopy 2007;23(04):340-346. Doi: 10.1016/j.arthro.2007.01.005

10 Sugaya H, Maeda K, Matsuki K, Moriishi J. Repair integrity and functional outcome after arthroscopic double-row rotator cuff repair. A prospective outcome study. J Bone Joint Surg Am 2007; 89(05):953-960. Doi: 10.2106/JBJS.F.00512

11 Wolf EM, Pennington WT, Agrawal V. Arthroscopic rotator cuff repair: 4- to 10-year results. Arthroscopy 2004;20(01):5-12. Doi: 10.1016/j.arthro.2003.11.00

12 Galatz LM, Ball CM, Teefey SA, Middleton WD, Yamaguchi K. The outcome and repair integrity of completely arthroscopically repaired large and massive rotator cuff tears. J Bone Joint Surg Am 2004;86(02):219-224. Doi: 10.2106/00004623-20040200000002

13 Boileau P, Brassart N, Watkinson DJ, Carles M, Hatzidakis AM, Krishnan SG. Arthroscopic repair of full-thickness tears of the supraspinatus: does the tendon really heal? J Bone Joint Surg Am 2005;87(06):1229-1240. Doi: 10.2106/JBJS.D.02035

14 Bishop J, Klepps S, Lo IK, Bird J, Gladstone JN, Flatow EL. Cuff integrity after arthroscopic versus open rotator cuff repair: a prospective study. J Shoulder Elbow Surg 2006;15(03):290-299. Doi: $10.1016 /$ j.jse.2005.09.017

15 Ellman H, Hanker G, Bayer M. Repair of the rotator cuff. End-result study of factors influencing reconstruction. J Bone Joint Surg Am 1986;68(08):1136-1144

16 Iannotti JP, Bernot MP, Kuhlman JR, Kelley MJ, Williams GR. Postoperative assessment of shoulder function: a prospective study of full-thickness rotator cuff tears. J Shoulder Elbow Surg 1996;5(06):449-457. Doi: 10.1016/S1058-2746(96)80017-6

17 Goutallier D, Postel JM, Gleyze P, Leguilloux P, Van Driessche S. Influence of cuff muscle fatty degeneration on anatomic and functional outcomes after simple suture of full-thickness tears. J Shoulder Elbow Surg 2003;12(06):550-554. Doi: 10.1016/ S1058-2746(03)00211-8

18 Lafosse L, Brozska R, Toussaint B, Gobezie R. The outcome and structural integrity of arthroscopic rotator cuff repair with use of the double-row suture anchor technique. J Bone Joint Surg Am 2007;89(07):1533-1541. Doi: 10.2106/JBJS.F.00305

19 Park MC, ElAttrache NS, Tibone JE, Ahmad CS, Jun BJ, Lee TQ. Part I: Footprint contact characteristics for a transosseous-equivalent rotator cuff repair technique compared with a double-row repair technique. J Shoulder Elbow Surg 2007;16(04):461-468. Doi: 10.1016/j.jse.2006.09.010

20 Ahmad CS, Stewart AM, Izquierdo R, Bigliani LU. Tendon-bone interface motion in transosseous suture and suture anchor rotator cuff repair techniques. Am J Sports Med 2005;33(11): 1667-1671. Doi: 10.1177/0363546505278252

21 Apreleva M, Özbaydar M, Fitzgibbons PG, Warner JJP. Rotator cuff tears: the effect of the reconstruction method on threedimensional repair site area. Arthroscopy 2002;18(05): 519-526. Doi: 10.1053/jars.2002.32930 
22 Kuroda S, Ishige N, Mikasa M. Advantages of arthroscopic transosseous suture repair of the rotator cuff without the use of anchors. Clin Orthop Relat Res 2013;471(11):3514-3522. Doi: 10.1007/s11999-013-3148-7

23 Black EM, Austin LS, Narzikul A, Seidl AJ, Martens K, Lazarus MD. Comparison of implant cost and surgical time in arthroscopic transosseous and transosseous equivalent rotator cuff repair. J Shoulder Elbow Surg 2016;25(09):1449-1456. Doi: 10.1016/j. jse.2016.01.003

24 Garofalo R, Castagna A, Borroni M, Krishnan SG. Arthroscopic transosseous (anchorless) rotator cuff repair. Knee Surg Sports Traumatol Arthrosc 2012;20(06):1031-1035. Doi: 10.1007/ s00167-011-1725-4

25 Seidl AJ, Lombardi NJ, Lazarus MD, et al. Arthroscopic Transosseous and Transosseous-Equivalent Rotator Cuff Repair: An Analysis of Cost, Operative Time, and Clinical Outcomes. Am J Orthop 2016;45(07):E415-E420

26 Kummer FJ, Hahn M, Day M, Meislin RJ, Jazrawi LM. A laboratory comparison of a new arthroscopic transosseous rotator cuff repair to a double row transosseous equivalent rotator cuff repair using suture anchors. Bull Hosp Jt Dis (2013) 2013;71(02):128-131

27 Park MC, Cadet ER, Levine WN, Bigliani LU, Ahmad CS. Tendon-tobone pressure distributions at a repaired rotator cuff footprint using transosseous suture and suture anchor fixation techniques. Am J Sports Med 2005;33(08):1154-1159. Doi: 10.1177/ 0363546504273053

28 Tauber M, Koller H, Resch H. Transosseous arthroscopic repair of partial articular-surface supraspinatus tendon tears. Knee Surg Sports Traumatol Arthrosc 2008;16(06):608-613. Doi: 10.1007/ s00167-008-0532-z

29 Yamaguchi K, Levine WN, Marra G, Galatz LM, Klepps S, Flatow EL. Transitioning to arthroscopic rotator cuff repair: the pros and cons. Instr Course Lect 2003;52:81-92

30 Fleega BA. Arthroscopic transhumeral rotator cuff repair: Giant needle technique. Arthroscopy 2002;18(02):218-223. Doi: 10.1053/jars.2002.30661

31 Black EM, Lin A, Srikumaran U, Jain N, Freehill MT. Arthroscopic transosseous rotator cuff repair: technical note, outcomes, and complications. Orthopedics 2015;38(05):e352-e358. Doi: 10.3928/01477447-20150504-50

32 Mahar AT, Moezzi DM, Serra-Hsu F, Pedowitz RA. Comparison and performance characteristics of 3 different knots when tied with 2 suture materials used for shoulder arthroscopy. Arthroscopy 2006;22(06):614.e1-614.e2. Doi: 10.1016/j.arthro.2006.02.005
33 Wüst DM, Meyer DC, Favre P, Gerber C. Mechanical and handling properties of braided polyblend polyethylene sutures in comparison to braided polyester and monofilament polydioxanone sutures. Arthroscopy 2006;22(11):1146-1153. Doi: 10.1016/j. arthro.2006.06.013

34 Rossouw DJ, McElroy BJ, Amis AA, Emery RJH. A biomechanical evaluation of suture anchors in repair of the rotator cuff. J Bone Joint Surg Br 1997;79(03):458-461

35 Goradia VK, Rochat MC, Kida M, Grana WA. Natural history of a hamstring tendon autograft used for anterior cruciate ligament reconstruction in a sheep model. Am J Sports Med 2000;28(01): 40-46

36 Pinczewski LA, Clingeleffer AJ, Otto DD, Bonar SF, Corry IS. Integration of hamstring tendon graft with bone in reconstruction of the anterior cruciate ligament. Arthroscopy 1997;13(05):641-643. Doi: 10.1016/S0749-8063(97)90194-8

37 Rodeo SA, Arnoczky SP, Torzilli PA, Hidaka C, Warren RF. Tendonhealing in a bone tunnel. A biomechanical and histological study in the dog. J Bone Joint Surg Am 1993;75(12):1795-1803

38 Weiler A, Peine R, Pashmineh-Azar A, Abel C, Südkamp NP, Hoffmann RFG. Tendon healing in a bone tunnel. Part I: Biomechanical results after biodegradable interference fit fixation in a model of anterior cruciate ligament reconstruction in sheep. Arthroscopy 2002;18 (02):113-123. Doi: $10.1053 /$ jars.2002.30656

39 Baums MH, Spahn G, Steckel H, Fischer A, Schultz W, Klinger H-M. Comparative evaluation of the tendon-bone interface contact pressure in different single- versus double-row suture anchor repair techniques. Knee Surg Sports Traumatol Arthrosc 2009;17 (12):1466-1472. Doi: 10.1007/s00167-009-0771-7

40 Lee TQ. Current biomechanical concepts for rotator cuff repair. Clin Orthop Surg 2013;5(02):89-97. Doi: 10.4055/cios.2013.5.2.89

41 Gupta H, Mishra P, Kataria H, et al. Optimal Angle of the Bone Tunnel for Avoiding Axillary Nerve Injuries During Arthroscopic Transosseous Rotator Cuff Repair: A Magnetic Resonance Imaging-Based Simulation Study. Orthop J Sports Med 2018;6 (11):2325967118806295. Doi: 10.1177/2325967118806295

42 Bronsnick D, Pastor A, Peresada D, Amirouche F, Solitro GF, Goldberg BA. Is Arthroscopic Transosseous Rotator Cuff Repair Strength Dependent on the Tunnel Angle? Orthop J Sports Med 2019;7 (06):2325967119848667. Doi: 10.1177/2325967119848667

43 Andres BM, Lam PH, Murrell GA. Tension, abduction, and surgical technique affect footprint compression after rotator cuff repair in an ovine model. J Shoulder Elbow Surg 2010;19(07):1018-1027. Doi: $10.1016 /$ j.jse.2010.04.005 